IEEC/CSM-99-61

hep-th/9906229

June 1999

\title{
On the concept of determinant for the differential operators of Quantum Physics
}

\author{
E. ELIZALDE円 \\ Consejo Superior de Investigaciones Científicas (CSIC), \\ Institut d'Estudis Espacials de Catalunya (IEEC), \\ Edifici Nexus 201, Gran Capità 2-4, 08034 Barcelona, Spain \\ and \\ Departament ECM i IFAE, Facultat de Física, \\ Universitat de Barcelona, Diagonal 647, 08028 Barcelona, Spain
}

\begin{abstract}
The concept of determinant for a linear operator in an infinite-dimensional space is addressed, by using the derivative of the operator's zeta-function (following Ray and Singer) and, eventually, through its zeta-function trace. A little play with operators as simple as $\pm I$ ( $I$ being the identity operator) and variations thereof, shows that the presence of a noncommutative anomaly (i.e., the fact that $\operatorname{det}(A B) \neq \operatorname{det} A \operatorname{det} B$ ), is unavoidable, even for commuting and, remarkably, also for almost constant operators. In the case of Dirac-type operators, similarly basic arguments lead to the conclusion - contradicting common lorethat in spite of being $\operatorname{det}(\not D+i m)=\operatorname{det}(\not D-i m)$ (as follows from the symmetry condition of the $\not D$-spectrum), it turns out that these determinants may not be equal to $\sqrt{\operatorname{det}\left(\not D^{2}+m^{2}\right)}$, simply because $\operatorname{det}[(\not D+i m)(\not D-i m)] \neq \operatorname{det}(\not D+i m) \operatorname{det}(\not D-i m)$. A proof of this fact is given, by way of a very simple example, using operators with an harmonic-oscillator spectrum and fulfilling the symmetry condition. This anomaly can be physically relevant if, in addition to a mass term (or instead of it), a chemical potential contribution is added to the Dirac operator.
\end{abstract}

PACS: 02.30.Lt, 02.30.Gp, 02.30.Tb

${ }^{1}$ E-mail: elizalde@ieec.fcr.es eli@ecm.ub.es http://www.ieec.fcr.es/cosmo-www/eli.htm 


\section{Introduction}

Many fundamental calculations of Quantum Field Theory reduce, in essence, to the computation of the determinant of some operator. One could even venture to say that, at one-loop order, any such theory reduces to a theory of determinants. The operators involved are 'differential' ones, as the normal physicist would say. In fact, properly speaking, they are pseudodifferential operators $(\Psi D O)$, that is, in loose terms 'some analytic functions of differential operators' (such as $\sqrt{1+D}$ or $\log (1+D)$, but not! $\log D)$. This is explained in detail in Refs. [四]- 3 .

Important as the concept of determinant of a differential or $\Psi$ DO may be for theoretical physicists (in view of what has just been said), it is surprising that this seems not to be a subject of study among function analysts or mathematicians in general. This statement must be qualified: I am specifically refering to determinants that involve in its definition some kind of regularization, very much related to operators that are not traceclass. This piece of calculus - always involving regularization - falls outside the scope of the standard disciplines and even many physically oriented mathematicians know little or nothing about it. In a sense, the subject has many things in common with that of divergent series but has not been so groundly investigated and lacks any reference comparable to the very beautiful book of Hardy [4]. Actually, from this general viewpoint, the question of regularizing infinite determinants was already addressed by Weierstrass in a way that, although it has been pursued by some theoretical physicists with success, is not without problems — as a general method - since it ordinarily leads to non-local contributions that cannot be given a physical meaning in QFT. We should mention, for completion, that there are, since long ago, well stablished theories of determinants for degenerate operators, for traceclass operators in the Hilbert space, Fredholm operators, etc. [5] but, again, these definitions of determinant do not fulfill all the needs mentioned above which arise in QFT.

Any high school student knows what a determinant is, in simple words, or at least how to calculate the determinant of a $3 \times 3$ matrix (and some of them, even that of a $4 \times 4$ one). But many one prominent mathematician will answer the question: What is your favourite definition of determinant of a differential operator? with: I don't have any, or: These operators don't have determinants! An even more 'simple' question I dare to ask the reader (which she/he may choose to ask to some other colleague on its turn) is the following: What is the value of the determinant of minus the identity operator in an infinite dimensional space? Followed by: And that of the determinant $\prod_{n \in N}(-1)^{n}$ ? Is it actually equal to the 
product of the separate determinants of the plus 1 s and of the minus $1 s$ ?

In this contribution I will point out to specific situations, some of them having become common lore already and other that have appeared recently in the literature, concerning the concept of determinant in QFT, and I will try to give 'reasonable' answers to questions such as the last ones.

\section{Infinite series and (almost) trivial determinants}

The mathematical theory of divergent series has been very fruitful in taming the infinites that have appeared in QFT, from the very begining of its conception. Its role is very essential, at least in the first stage of the regularization/renormalization procedure. Euler and Borel summation methods, and analytic continuation techniques are there commonly used. But some difficulties exist that are inherent to the theory of divergent series (see, for instance, [4]). One of them is the well known fact that, sometimes, by using different schemes, different results are obtained. In a well posed physical situation, the 'right' one can then only be choosen after experimental validation. Another problem is to understand, in physical terms, what you are doing, while performing say an analytic continuation from one region of the complex plane to another [ [6]. This has prevented e.g.the zeta function regularization procedure from getting general aceptance among common physicists.

The situation concerning infinite determinants is even worse, in a sense. There is no book on the subject to be compared, for instance, with the above mentioned one by Hardy and we see every day that dubious manipulations are being performed at the level of the eigenvalues, that are then translated to the determinant itself and elevated sometimes to the cathegory of standard results - when not of lore theorems. The first problem is the definition of the

determinant itself. Let me quote in this respect from a recent paper by E. Witten [7]: The determinant of the Dirac operator is defined roughly as

$$
\operatorname{det} \mathcal{D}=\prod_{i} \lambda_{i}
$$

where the infinite product is regularized with (for example) zeta function or Pauli-Villars regularization. The zeta function definition of the determinant

$$
\operatorname{det}_{\zeta} \mathcal{D}=\exp \left[-\zeta_{\mathcal{D}}{ }^{\prime}(0)\right]
$$

is maybe the one that has more firm mathematical grounds [8]. In spite of starting from the identity: $\log$ det $=\operatorname{tr} \log$, it is known to develop the so called multiplicative anomaly: 
the determinant of the product of two operators is not equal, in general, to the product of the determinants (even if the operators commute!). This happens already with very simple operators (as two one-dimensional harmonic oscillators only differing in a constant term, Laplacians plus different mass terms, etc.). It may look incredible, at first sight, from the tr log property and the additivity of the trace, but we must just take into account that the zeta trace is no ordinary trace (for it involves regularization), namely:

$$
\operatorname{tr}_{\zeta} \mathcal{D}=\zeta_{\mathcal{D}}(-1)
$$

so that $\operatorname{tr}_{\zeta}(A+B) \neq \operatorname{tr}_{\zeta} A+\operatorname{tr}_{\zeta} B$, in general. Not to understand this has originated a considerable amount of errors in the specialized literature - falsely attributed to missfunctions of the rigorous and elegant zeta function method!

As an example, consider the following commuting linear operators in an infinite-dimensional space, given in diagonal form by:

$$
O_{1}=\operatorname{diag}(1,2,3,4, \ldots), \quad O_{2}=\operatorname{diag}(1,1,1,1, \ldots) \equiv I
$$

and their sum

$$
O_{1}+O_{2}=\operatorname{diag}(2,3,4,5, \ldots)
$$

The corresponding $\zeta$-traces are easily obtained:

$$
\begin{aligned}
& \operatorname{tr}_{\zeta} O_{1}=\zeta_{R}(-1)=-\frac{1}{12}, \quad \operatorname{tr}_{\zeta} O_{2}=\zeta_{R}(0)=-\frac{1}{2}, \\
& \operatorname{tr}_{\zeta}\left(O_{1}+O_{2}\right)=\zeta_{R}(-1)-1=-\frac{13}{12}
\end{aligned}
$$

the last trace having been calculated according to the rules of infinite series summation (see e.g., Hardy 团). We observe that

$$
\operatorname{tr}_{\zeta}\left(O_{1}+O_{2}\right)-\operatorname{tr}_{\zeta} O_{1}-\operatorname{tr}_{\zeta} O_{2}=-\frac{1}{2} \neq 0
$$

If this happens in such simple situation, involving the identity operator, one can easily imagine that any precaution one can take in manipulating infinite sums might turn out to be insufficient. Moreover, since the multiplicative anomaly - as has been pointed out before - originates precisely in the failure of this addition property for the regularized trace, we can already guess that it also can show up in very simple situations, as will now be proven, in fact. The appearance of the multiplicative anomaly prevents, in particular, naive 
manipulations with the eigenvalues in the determinant, as reorderings and splittings, what a number of physicists seem not to be aware of.

For warming up, let us calculate some simple determinants with the zeta function method. To start with, take

$$
\Delta_{1}=\prod_{n=1}^{\infty} n
$$

We have

$$
\zeta_{1}(s)=\sum_{n=1}^{\infty} n^{-s}=\zeta_{R}(s), \quad \zeta_{R}^{\prime}(0)=-\frac{1}{2} \log (2 \pi)
$$

so that

$$
\Delta_{1}=\exp \left[\frac{1}{2} \log (2 \pi)\right]=\sqrt{2 \pi}
$$

a nice result. In the same way, we obtain

$$
\Delta_{2}=\prod_{n=1}^{\infty} n^{-1}=\frac{1}{\sqrt{2 \pi}}
$$

- from $\zeta_{2}(s)=\zeta_{R}(-s)$ - as should be expected.

Let us now consider the apparently more simple case:

$$
\Delta_{3}=\prod_{n=1}^{\infty} \lambda
$$

This poses a problem to the zeta function method, which must be modified somehow to cope with such situation. In fact, the corresponding zeta function,

$$
\zeta_{3}(s)=\sum_{n=1}^{\infty} \lambda^{-s}
$$

has no abscissa of convergence in the complex plane (since the sequence of eigenvalues is neither increasing nor decreasing. This can be, however, naturally solved as follows: by taking logarithms (what is inherent with the definition of the zeta function method) and using again the rules for infinite series, it is plain that the result is

$$
\log \Delta_{3}=\log \lambda \sum_{n=1}^{\infty} 1=-\frac{1}{2} \log \lambda
$$

where the factor in front of $\log \lambda$ may be interpreted as the 'zeta measure' of the set of positive natural numbers, thus

$$
\Delta_{3}=\prod_{n=1}^{\infty} \lambda=\lambda^{-\frac{1}{2}}
$$


This leads, in particular, to the following results:

$$
\Delta_{4}=\prod_{n=1}^{\infty} 1=1^{-\frac{1}{2}}= \pm 1
$$

for the determinant of the identity operator, $I$, and

$$
\Delta_{5}=\prod_{n=1}^{\infty}(-1)=(-1)^{-\frac{1}{2}}=\mp i
$$

for the determinant of the operator $-I$. As it seems clear that the determinant of the identity operator should be 1, this tells us (by choice everywhere of the same determination of the logarithm in the complex plane) that the determinant of $-I$ is $-i$ and, that of $\lambda I$, the inverse of the corresponding square root of $\lambda$ in (15). Notice that, in this way, we are starting to build up a set of consistency rules that are reminiscent, in some manner, of the corresponding rules for infinite series [四]. More than this, by use of the logarithm, all the ordinary rules for infinite series are appliable to the series of logs of eigenvalues, in particular, the ones concerning multiplication by a common factor (used before already), or of splitting out a finite number of first terms from the series (that is to say, a finite number of first factors from the determinant). However, the splitting of an infinite number of terms - or of the whole series into two - is not allowed in general. That is, again, the lesson we have learned from the existence of the multiplicative anomaly of the determinant when evaluated by the zeta function procedure. An additional comment is in order: in dealing with infinite series we always take logarithms, and this introduces an ambiguity in the zeta function definition of the determinant. This fact is well known [9, 10] and is common to other regularization methods (as Pauli-Villars'), under different disguises. It can duely be taken care of by sticking to one and the same determination of the logarithm during the whole calculation.

An apparent problem — or virtue perhaps? - of the zeta function definition of infinite determinants is posed by the following example. It turns out that the determinants

$$
\Delta_{6}=\prod_{n=1}^{\infty}(-1)^{2 n+1}, \quad \Delta_{7}=\prod_{n=1}^{\infty}(-1)^{4 n+1}, \quad \ldots
$$

and $\Delta_{5}$ are all different. This originates in

$$
\begin{aligned}
& \zeta_{6}(-1)=2 \zeta_{H}(-1,1 / 2)-1=-B_{2}(1 / 2)-1=-\frac{11}{12}, \\
& \zeta_{7}(-1)=4 \zeta_{H}(-1,1 / 4)-1=-2 B_{2}(1 / 4)-1=-\frac{23}{24}, \quad \ldots
\end{aligned}
$$


and can be interpreted as due to the change of the 'zeta measure' of the number of factors in the product leading to the determinant. We could try to avoid this problem by sticking always to the most simple characterization of the eigenvalues series (in this case $-1,-1,-1, \ldots$, any reference to $n$ being superfluous). Things are, regretfully, not that simple. Consider the determinants $\Delta_{4}, \Delta_{5}$, and

$$
\Delta_{8}=\prod_{n=1}^{\infty}(-1)^{n},
$$

and try to make compatible the apparently obvious fact that:

$$
\Delta_{8}=\sqrt{\Delta_{4} \Delta_{5}}
$$

The determinant $\Delta_{8}$ can be obtained in three different ways, that yield the same result.

(i) We have, through the corresponding zeta function,

$$
\begin{aligned}
& \zeta_{8}(s)=\sum_{n=1}^{\infty}(-1)^{-n s}=\frac{1}{(-1)^{s}-1}, \\
& \zeta_{8}^{\prime}(s)=\log (-1)\left[\frac{1}{\pi^{2} s^{2}}+\frac{1}{12}+\mathcal{O}(s)\right], \\
& \Delta_{8}=\exp \left[-\left.\zeta_{8}^{\prime}(0)\right|_{r e g}\right]=(-1)^{-1 / 12} .
\end{aligned}
$$

(ii) On the other hand, taking logs as before, from the zeta function measure of the set of exponents, we get

$$
\begin{aligned}
& \log \Delta_{8}=\log (-1) \sum_{n=1}^{\infty} n=\log (-1) \zeta_{R}(-1), \\
& \Delta_{8}=(-1)^{\zeta_{R}(-1)}=(-1)^{-1 / 12} .
\end{aligned}
$$

(iii) Also, we may instead choose to take derivatives, term by term, in the first expression for the zeta function

$$
\begin{aligned}
& \zeta_{8}(s)=\sum_{n=1}^{\infty}(-1)^{-n s} \\
& \zeta_{8}^{\prime}(s)=-\sum_{n=1}^{\infty} n(-1)^{-n s} \log (-1), \quad \zeta_{8}^{\prime}(0)=-\log (-1) \sum_{n=1}^{\infty} n=\frac{\log (-1)}{12} \\
& \Delta_{8}=\exp \left[-\zeta_{8}^{\prime}(0)\right]=(-1)^{-1 / 12} .
\end{aligned}
$$

Remarkably enough, in all three cases we obtain the same result for this determinant. Let us now try to fulfill the factorization condition (21). We have:

$$
\begin{aligned}
& \Delta_{4}=\prod_{n=1}^{\infty} 1=1^{-1 / 2}= \pm 1, \quad \Delta_{5}=\prod_{n=1}^{\infty}(-1)=(-1)^{-1 / 2}=\mp i, \\
& \Delta_{8}=\prod_{n=1}^{\infty}(-1)^{n}=(-1)^{-1 / 12}=\sqrt{\Delta_{4} \Delta_{5}}=1^{-1 / 4}(-1)^{-1 / 4} .
\end{aligned}
$$


The only way to fulfill this property (21) is to accept that:

$$
\prod_{n=1}^{\infty} 1=-1 \quad ! !
$$

On the contrary, if we insists (as almost everybody would agree on) that the determinant of the identity is 1 , then we must give up the property that the determinant of the alternating series of eigenvalues $1,-1,1,-1, \ldots$ is equal to the subdeterminant product of the $1 \mathrm{~s}$, times the subdeterminant product of the $-1 \mathrm{~s}$. This is the most simple reflection one could ever have expected to obtain of the multiplicative anomaly of the determinant!

If we choose to preserve, at any price, the multiplication property of the determinant and give sense to the strange fact that det $I=-1$, then we do attain compatibility in Eqs. (25) by setting:

$$
\prod_{n=1}^{\infty} 1=e^{i \pi}, \quad \prod_{n=1}^{\infty}(-1)=e^{i \pi / 2}, \quad \prod_{n=1}^{\infty}(-1)^{n}=e^{3 i \pi / 4}, \quad \prod_{n=1}^{\infty}(-1)^{4 n}=e^{3 i \pi}, \ldots
$$

All these are compatible zeta function definitions of the determinant (they can be fixed from acceptable roots of 1 or -1 as given by the zeta function exponents) satisfying the multiplication rule. However, it is easy to see that this process cannot go for ever (and thus eliminate the anomaly): the following dets cannot possibly fulfill the multiplicative property:

$$
\prod_{n=1}^{\infty}(-1)^{2 n+1}=(-1)^{-11 / 12} \neq \prod_{n=1}^{\infty}(-1)^{2 n} \prod_{n=1}^{\infty}(-1)
$$

But maybe this is asking too much, as has been observed before, in which case we are still left with the compatible (albeit really weird) choice (27).

Anyhow, it is easy to check that we do not find problems in factorizations like

$$
\prod_{n=1}^{\infty}(-n)=\prod_{n=1}^{\infty}(-1) \prod_{n=1}^{\infty} n,
$$

since

$$
\begin{aligned}
& \zeta(s)=(-1)^{-s} \zeta_{R}(s), \quad \zeta^{\prime}(0)=-\frac{1}{2} \log (-1)-\frac{1}{2} \log (2 \pi), \\
& \prod_{n=1}^{\infty}(-n)=e^{-\zeta^{\prime}(0)}=(-1)^{-1 / 2} \sqrt{2 \pi}=\prod_{n=1}^{\infty}(-1) \prod_{n=1}^{\infty} n
\end{aligned}
$$

or in the more involved one

$$
\prod_{n=1}^{\infty}(-1)^{n} n=\prod_{n=1}^{\infty}(-1)^{n} \prod_{n=1}^{\infty} n
$$


because

$$
\begin{aligned}
& \zeta(s)=\sum_{n=1}^{\infty}(-1)^{-n s} n^{-s}=\Phi\left((-1)^{-s}, s\right), \quad \zeta^{\prime}(0)=\frac{i \pi}{12}+\Phi^{\prime}(0,1)=\frac{i \pi}{12}-\frac{1}{2} \log (2 \pi), \\
& \prod_{n=1}^{\infty}(-1)^{n} n=e^{-\zeta^{\prime}(0)}=(-1)^{-1 / 12} \sqrt{2 \pi}=\prod_{n=1}^{\infty}(-1)^{n} \prod_{n=1}^{\infty} n
\end{aligned}
$$

being $\Phi$ the polylogarithm function. The factorization of the determinants holds here again, in the zeta function prescription, and this fact does not seem to be that immediate, in view of the last calculation as compared with what we had before.

All the above considerations may sound rather trivial, but actually they are not, and should be carefully taken into account before proceding with the sort of manipulations of the eigenvalues and splittings of determinants that pervade the specialized literature.

\section{The multiplicative anomaly for Dirac type operators}

Consider the ordinary Dirac equation for a massive spinor

$$
(\not D+i m) \psi=0
$$

Usually, the determinant of the Dirac operator acting in this equation is obtained by using the following argument (see, e.g., [1] ):

$$
\operatorname{det}(\not D+i m)=\operatorname{det}(\not D-i m)=\left[\operatorname{det}\left(\not D^{2}+m^{2}\right)\right]^{1 / 2}
$$

This comes about from the fact that the spectrum of the Dirac massless operator $\not D$ has the following property: if $\lambda$ belongs to the spectrum, then so does $-\lambda$, that is immediately obtained by use of the $\gamma_{5}$ operator. Then, it turns out that the first det in Eq. (34) is a product of pairs of the form:

$$
(\lambda+i m)(-\lambda+i m)=(-\lambda-i m)(\lambda-i m)=(\lambda-i m)(-\lambda-i m)
$$

the last being the pairs appearing in the second det of Eq. (34). This is an algebraic argument, but there is also the corresponding geometric one, trivial after representing the spectral points in the complex plane. Thus, the first equality in (34) is proven, and the second seems obvious.

However, due to the existence of the multiplicative anomaly for infinite determinants [namely, the fact that, in $\operatorname{general}, \operatorname{det}(A B) \neq(\operatorname{det} A)(\operatorname{det} B)]$, all these formulas, obtained by 'simple' manipulation of the eigenvalues, must be set under suspicion and are in need of a 
rigorous check. Concerning Eq. (34, the second equality cannot be taken for granted, since it may turn out that

$$
\operatorname{det}[(\not D+i m)(\not D-i m)] \neq \operatorname{det}(\not D+i m) \operatorname{det}(\not D-i m)
$$

We will show below that this is indeed the case, in a very simple, parallel example completely under control - that uses as operator some square root of the harmonic oscillator.

Indeed, consider the square root of the harmonic oscillator obtained by Delbourgo in Ref. [12]. This example has potentially some interesting physical applications, for it is well known that a fermion in an external constant electromagnetic field has a similar spectrum (Landau spectrum). Exactly in the same way as when going from the Klein-Gordon to the Dirac equation and paying the same price of doubling the number of components (e.g., introducing spin), Delbourgo has constructed a model for which there exists a square root of its Hamiltonian, which is very close to the one for the harmonic oscillator. It is in fact different from the Dirac oscillator introduced by several other authors, corresponding to the minimal substitution $\vec{p} \rightarrow \vec{p}-i \alpha \vec{r}$. The main difference lies in the introduction now of the parity operator, $Q$. Whereas creation and destruction operators for the harmonic oscillator, $a^{ \pm}=P \pm i X$, are non-hermitian, the combinations $\mathcal{D}^{ \pm}=P \pm i Q X$ are hermitian and

$$
H^{ \pm} \equiv\left(\mathcal{D}^{ \pm}\right)^{2}=P^{2}+X^{2} \mp Q=2 H_{\mathrm{OSC}} \mp Q
$$

Notice that the parity term commutes with $H_{\mathrm{OSc}}$. Doubling the components $\left(\sigma_{i}\right.$ are the Pauli matrices)

$$
P \rightarrow-i \sigma_{1} \frac{\partial}{\partial x}, \quad X \rightarrow \sigma_{1} x, \quad Q \rightarrow \sigma_{2}
$$

the operators $\mathcal{D}^{ \pm}$are represented by

$$
\mathcal{D}^{ \pm} \rightarrow-i \sigma_{1} \frac{\partial}{\partial x} \pm \sigma_{3} x
$$

In the sequel, we will only consider the operator $\mathcal{D} \equiv \mathcal{D}^{+}$. It has for eigenfunctions and eigenvalues, respectively,

$$
\begin{gathered}
\psi_{n}^{ \pm}(x)=\frac{-i e^{-x^{2} / 2}}{\sqrt{2^{n+1}(n-1) ! \sqrt{\pi}}}\left(\begin{array}{c}
-i\left[H_{n-1}(x) \pm H_{n}(x) / \sqrt{2 n}\right] \\
{\left[H_{n-1}(x) \mp H_{n}(x) / \sqrt{2 n}\right]}
\end{array}\right), \quad \lambda_{n}= \pm \sqrt{2 n}, n \geq 1, \\
\psi_{0}(x)=\frac{e^{-x^{2} / 2}}{\sqrt{2 \sqrt{\pi}}}\left(\begin{array}{c}
1 \\
i
\end{array}\right), \quad \lambda_{0}=0,
\end{gathered}
$$


where the $H_{n}(x)$ are Hermite polinomials.

The two operators we shall consider for the calculation of the anomaly are $A=\mathcal{D}+V$ and $B=\mathcal{D}-V, V$ being a real, constant potential with $|V|<\sqrt{2}$, that goes multiplied with the identity matrix in the two (spinorial) dimensions (omitted here). Obviously, this $V$ is to be identified with the mass $m$, to make contact with the case of the Dirac equation that we had at the begining.

Notice that $\mathcal{D}+V$ and $\mathcal{D}-V$ are hermitian, commuting operators. The multiplicative anomaly is defined as

$$
a(A, B)=\log \operatorname{det}(A B)-\log \operatorname{det} A-\log \operatorname{det} B=\zeta_{A}{ }^{\prime}(0)+\zeta_{B}{ }^{\prime}(0)-\zeta_{A B}{ }^{\prime}(0) .
$$

The zeta function for the operator $\mathcal{D}$ reads

$$
\zeta_{\mathcal{D}}(s)=\sum_{i} \lambda_{i}^{-s}=\sum_{n=1}^{\infty}\left[1+(-1)^{-s}\right](\sqrt{2 n})^{-s}=\left[1+(-1)^{-s}\right] 2^{-s / 2} \zeta_{R}(s / 2),
$$

$\zeta_{R}(s)$ being the usual Riemann zeta function, which has a simple pole at $s=1$. The zeta function for the operators $\mathcal{D} \pm V$ is (see, for instance, [13])

$$
\zeta_{\mathcal{D} \pm V}(s)=\sum_{i}\left(\lambda_{i} \pm V\right)^{-s}=\zeta_{\mathcal{D}}(s)+\sum_{n=1}^{\infty} \frac{(\mp V)^{n} \Gamma(n+s)}{n ! \Gamma(s)} \zeta_{\mathcal{D}}(n+s) .
$$

Finally, the zeta function for the operator $(\mathcal{D}+V)(\mathcal{D}-V)=\mathcal{D}^{2}-V^{2}$ is given by:

$$
\zeta_{\mathcal{D}^{2}-V^{2}}(s)=\zeta_{\mathcal{D}}(2 s)+\sum_{n=1}^{\infty} \frac{V^{2 n} \Gamma(n+s)}{n ! \Gamma(s)} \zeta_{\mathcal{D}}(2 n+2 s)
$$

We see that the anomaly in this simple case can be obtained in terms of the derivatives of the Riemann zeta function. The important fact is that it turns out to be non-zero:

$$
a(\mathcal{D}+V, \mathcal{D}-V)=2 V^{2}
$$

We should point out that this result can be obtained from the Wodzicki formula for the anomaly, even if we are working in a non-compact manifold 13. We thus have a very simple example of the presence of a non-trivial anomaly for operators of degree one in a space of dimension one (spinorial, however).

One can argue that a mass term will be absorbed by renormalization and will finally yield no physical contribution. Notice, however, that the situation is much more general than the specific case considered here, which, however, even in its simplicity already accounts for any kind of terms not depending on the space-time coordinates. Thus $V$ can represent - aside from a mass term $i m$ - a constant magnetic field, a finite temperature term, or a chemical potential. Anomalous contributions of this kind cannot be absorbed by renormalization (see, e.g., [14]) and can acquire a direct physical meaning [13, 15]. 


\section{Acknowledgments}

The author is indebted with Andreas Wipf and Sergio Zerbini for enlightening discussions and with the members of the Institutes of Theoretical Physics of the Universities of Jena and Trento, where the main part of this work was done, for warm hospitality. This investigation has been supported by DGICYT (Spain), project PB96-0925, by CIRIT (Generalitat de Catalunya), by the Italian-Spanish program INFN-CICYT, and by the German-Spanish program Acciones Integradas, project HA1997-0053.

\section{References}

[1] E. Elizalde, Commun. Math. Phys. 198, 83 (1998).

[2] A.P. Calderón and A. Zygmund, Am. J. Math. 79, 801 (1957); Studia Math. 20, 171 (1961); A.P. Calderón and R. Vaillancourt, Proc. Nat. Acad. Sci. U.S.A. 69, 1185 (1972).

[3] L. Hörmander, The analysis of partial differential operators, Vols I-IV (Springer, Berlin, 1983-85); F. Treves, Introduction to pseudodifferential and Fourier integral operators, Vols. I and II (Plenum, New York, 1980); M.E. Taylor, Pseudodifferential operators (Princeton University Press, Princeton, 1981); H. Lawson and M.L. Michelsohn, Spin geometry (Princeton University Press, Princeton, 1989).

[4] G.H. Hardy, Divergent Series (Oxford University Press, Oxford, 1949).

[5] T. Kato, Perturbation Theory for Linear Operators (Springer, Berlin, 1980).

[6] E. Elizalde, Ten physical applications of spectral zeta functions (Springer, Berlin, 1995); E. Elizalde, S.D. Odintsov, A. Romeo, A.A. Bytsenko and S. Zerbini, Zeta regularization techniques with applications (World Sci., Singapore, 1994).

[7] E. Witten, Supersymmetric index of three-dimensional gauge theory, hep-th/9903005; E. Witten, Commun. Math. Phys. 121, 351 (1989).

[8] D.B. Ray, Adv. in Math. 4, 109 (1970); D.B. Ray and I.M. Singer, Adv. in Math. 7, 145 (1971); Ann. Math. 98, 154 (1973). 
[9] M. Kontsevich and S. Vishik. Functional Analysis on the Eve of the 21st Century. volume 1, 173-197, (1993).

[10] D. Seminara, Parity and large gauge invariance in thermal QED 3 , hep-th/9812137; S. Deser, L. Griguolo and D. Seminara, Phys. Rev. D57, 7444 (1998).

[11] D.G.C. McKeon and C. Schubert, Phys. Lett. B440, 101 (1998).

[12] R. Delbourgo, A square root of the harmonic oscillator. University of Tasmania Preprint hep-th/9503056 (1995).

[13] E. Elizalde, G. Cognola and S. Zerbini, Nucl. Phys. 532, 407 (1998).

[14] J.J. McKenzie-Smith and D.J. Toms, Phys. Rev. D58, 105001 (1998).

[15] E. Elizalde, A. Filippi, L. Vanzo and S. Zerbini, Phys. Rev. D57, 7430 (1998); Is the multiplicative anomaly dependent on the regularization?, hep-th/9804071 (1998); Is the multiplicative anomaly relevant?, hep-th/9804072 (1998); J. S. Dowker, On the relevance of the multiplicative anomaly, hep-th/9802029 (1998); T. S. Evans, Regularization schemes and the multiplicative anomaly, hep-th/9803184 (1998). 